\title{
¿Explicit Filtering and Reconstruction to Reduce Grid Dependence in Convective Boundary Layer Simulations Using WRF-LES
}

\author{
JASON S. SIMON \\ University of California, Berkeley, Berkeley, California \\ BOWEN ZHOU \\ Key Laboratory for Mesoscale Severe Weather, Ministry of Education, and School of Atmospheric Sciences, \\ Nanjing University, Nanjing, China \\ JEFFREY D. MIROCHA \\ Lawrence Livermore National Laboratory, Livermore, California \\ Fotini KATOPODES CHOW \\ University of California, Berkeley, Berkeley, California
}

(Manuscript received 10 June 2018, in final form 12 December 2018)

\begin{abstract}
As model grid resolutions move from the mesoscale to the microscale, turbulent structures represented in atmospheric boundary layer simulations change dramatically. At intermediate resolutions, the so-called gray zone, turbulent motions are not resolved accurately, posing a challenge to numerical simulations. The representation of turbulence is also highly sensitive to the choice of closure model. Here, we examine explicit filtering and reconstruction in the gray zone as a technique to better represent atmospheric turbulence. The convective boundary layer is simulated using the Weather Research and Forecasting (WRF) Model with horizontal resolutions ranging from $25 \mathrm{~m}$ to $1 \mathrm{~km}$. Four large-eddy simulation (LES) turbulence models are considered: the Smagorinsky model, the TKE-1.5 model, and two versions of the dynamic reconstruction model (DRM). The models are evaluated by their ability to produce consistent mean potential temperature profiles, heat and momentum fluxes, velocity fields, and turbulent kinetic energy spectra as the grids become coarser. The DRM, a mixed model that uses an explicit filtering and reconstruction technique to account for resolvable subfilter-scale (RSFS) stresses, performs very well at resolutions of $500 \mathrm{~m}$ and $1 \mathrm{~km}$ without any special tuning, whereas the Smagorinsky and TKE-1.5 models produce heavily grid-dependent results.
\end{abstract}

\section{Introduction}

Mesoscale atmospheric models used for studying atmospheric dynamics and for numerical weather prediction (NWP) historically use horizontal resolutions that are $\mathscr{O}(1-10) \mathrm{km}$, which are much larger than the dominant length scale of turbulence in the atmosphere. Since turbulent motions in the atmosphere cannot be

Denotes content that is immediately available upon publication as open access.

Corresponding author: Fotini Katopodes Chow, tinakc@ berkeley.edu resolved at such coarse horizontal resolutions, their effects are parameterized. The parameterizations used to represent the impact of turbulence on the mean flow are typically based on Reynolds averaging, which uses an ensemble average to separate mean and fluctuating quantities, of the Navier-Stokes equations:

$$
\frac{\partial \bar{u}_{i}}{\partial t}+\frac{\partial \bar{u}_{i} \bar{u}_{j}}{\partial x_{j}}=-\frac{1}{\rho} \frac{\partial \bar{p}}{\partial x_{i}}-\frac{\partial \tau_{i j}}{\partial x_{j}}+\bar{F}_{i},
$$

where $x_{i}$ represent $x, y, z$ coordinates, $\bar{u}_{i}$ are the mean velocities, $p$ is pressure, $\rho$ is density, and molecular viscosity effects are neglected. The effect of turbulent motions is contained in the Reynolds stress term, $\tau_{i j}=\overline{u_{i}^{\prime} u_{j}^{\prime}}$, where 
$u_{i}^{\prime}$ is the fluctuating velocity field. Most mesoscale models use a planetary boundary layer (PBL) scheme to parameterize ensemble-averaged turbulent mixing in the boundary layer because it is assumed that PBL eddies cannot be resolved on a mesoscale grid.

In large-eddy simulation (LES), the large, energycontaining scales are resolved while smaller scales are parameterized. A turbulence closure is used to model the subfilter-scale (SFS) motions that are smaller than a selected spatial filter width. With LES, the overbar in Eq. (1) indicates a spatial filter and $\tau_{i j}=\overline{u_{i} u_{j}}-\bar{u}_{i} \bar{u}_{j}$ is now the SFS turbulent stress. Commonly, there is no explicit filter defined and the grid combined with the effects of the discretization schemes are considered to be an implicit filter. This implicit filter is effectively wider than the grid's Nyquist limit, the smallest wavelength that can be resolved on a given grid. In contrast, defining an explicit filter allows reconstruction of resolvable subfilter-scale (RSFS) motions, enabling more accurate representation of fine scales (Gullbrand and Chow 2003; Chow et al. 2005). Furthermore, by providing stresses from the missing scales, explicit filtering and reconstruction enable a more accurate representation of the more important energetic scales, as discussed further below.

Given the fine mesh spacing required to resolve turbulence, LES has historically been limited to idealized atmospheric studies and small-scale engineering applications where domain sizes are small enough to be manageable with the available computing power. As computational resources expand, users are now pushing mesoscale models to much higher resolutions, allowing for a more accurate representation of many properties (e.g., terrain, land-use, urban effects) as well as atmospheric features such as microfronts and terrain-induced flows, for example. As such, many NWP models have adopted options for LES, enabling microscale simulations. There are many recent examples in the literature of forecasting research reaching kilometer and finer scales for varying applications, such as wind energy (Liu et al. 2011), scalar dispersion (Klose and Shao 2013; Taylor et al. 2016), cloud modeling (Zhu et al. 2010) and use of high-resolution surface data (Garcia et al. 2014). Longer-term or operational simulations are also moving to higher resolution. This is problematic from a boundary layer turbulence perspective, however, because kilometer-scale grids can be on the same order as the size of the largest turbulent eddies in the atmospheric boundary layer (ABL) [e.g., organized thermals in the convective boundary layer (CBL)]. This leaves forecasters with a range of resolutions that are too coarse for LES and too fine for mesoscale turbulence modeling. This gap in turbulence parameterizations is often referred to as the gray zone or the terra incognita (Wyngaard 2004). In the atmospheric boundary layer of depth $z_{i}$, it is often suggested that the gray zone consists of resolutions where $\Delta_{x, y} \sim z_{i}$ (though this definition will be examined here).

The gray zone has been the subject of many recent studies from multiple perspectives. Honnert et al. (2011) conducted an extensive study on the balance between resolved and subgrid-scale (SGS) turbulent kinetic energy (TKE) at different resolutions. Grid-dependent dynamics, in the form of unphysically large convective cells, have been documented using multiple numerical models and turbulence closures (Lean et al. 2008; Piotrowski et al. 2009; Ching et al. 2014; Zhou et al. 2014; Shi et al. 2018).

Beare (2014) conducted an LES study on the gray zone for the CBL and proposed a gray zone criterion based on a dissipation length scale diagnosed from the second moment of the TKE. Efstathiou and Beare (2015) explored the gray zone of the Smagorinsky turbulence model for a variety of shear and surface heating combinations, observing a delay in the onset of resolved TKE in the gray zone and that profiles of resolved TKE become unrealistic when $\Delta_{x, y}>200 \mathrm{~m}$. Khani and Waite (2014, 2015) studied the Kraichnan, Smagorinsky and dynamic Smagorinsky models and found that different turbulence closures have different grid-spacing criteria for accurate simulations of stratified turbulence and proposed a gray zone criterion based on the buoyancy length scale. Multiple strategies for simulating the atmosphere in the gray zone have been proposed, including local grid refinement near the surface (Zhou et al. 2017, 2018), distinguishing between updrafts and downdrafts (Thuburn et al. 2018), and improving eddy-viscosity models to better adapt to gray-zone resolutions (Efstathiou et al. 2016; Kurowski and Teixeira 2018).

Here, we use the Weather Research and Forecasting (WRF) Model to examine the role of the turbulence closure model in LES on the behavior of CBL simulations in the gray zone. Four LES closure models are tested: the Smagorinsky model, the TKE-1.5 model, and the dynamic reconstruction model (DRM) with level-0 and level-2 reconstruction (see section 1c). Specifically, we study the performance of the DRM (Chow et al. 2005) as implemented in WRF (Kirkil et al. 2012). The DRM is built from an explicit filtering and reconstruction framework, which improves the representation of turbulent motions. Simulations of the CBL are performed at varying resolutions, in a process similar to Zhou et al. (2014) but using LES and at finer resolutions.

Our goal here is to examine the role of the turbulence closure model in the gray zone by examining mean profiles, the onset of convection, turbulence statistics, 
and velocity fields. In particular, it is hypothesized that the DRM can be helpful in reducing grid dependence in the gray zone by increasing the accuracy of the resolved scales and allowing for backscatter. The DRM has been shown to improve mean and turbulent profiles in multiple ABL regimes, including the neutral, stable, and stratocumulus-topped boundary layer when compared to eddy-viscosity models (Chow et al. 2005; Zhou and Chow 2011; Shi et al. 2018). In addition to exploring the performance of the DRM in detail, we proceed with the hypothesis that each closure model will behave differently in the gray zone. This means that the resolution at which the model transitions from well-resolved LES to the gray zone will be different depending on the closure model used.

\section{Turbulence closure models}

The first two turbulence models presented, the Smagorinsky and TKE-1.5 models, are eddy-viscosity models, which generally take the following form:

$$
\tau_{i j}=-2 K_{h, v} \bar{S}_{i j}
$$

where $\bar{S}_{i j}=1 / 2\left(\partial \bar{u}_{i} / \partial x_{j}+\partial \bar{u}_{j} / \partial x_{i}\right)$ is the resolved strainrate tensor and $K_{h, v}$ is the horizontal or vertical eddy viscosity.

Eddy-viscosity models are purely dissipative models and do not allow for any upscale transfer of energy from the SFS to the resolved scale. This may be acceptable behavior in the inertial subrange, but becomes less realistic as the grid's Nyquist limit approaches and passes the dominant energetic length scale of the CBL eddies. For an eddyviscosity model, $\tau_{i j}$ is often referred to as the SGS term despite commonly being used to represent the entire SFS. Here we will refer to $\tau_{i j}$ as the SFS stress for all models.

The DRM presented here uses explicit filtering and includes a scale-similarity component which allows for backscatter of energy from the SFS to the resolved scale (Zhou and Chow 2012). The DRM uses a dynamically calculated eddy viscosity to represent the effects of the SGS motions a la Germano et al. (1991).

\section{a. The Smagorinsky model}

In the WRF model (Skamarock et al. 2008), the threedimensional Smagorinsky model (Lilly 1962; Smagorinsky 1963; Lilly 1967) diagnoses the eddy-viscosity as

$$
K_{h, v}=C_{s}^{2} l_{h, v}^{2} \max \left[0,\left(\bar{S}_{i j} \bar{S}_{i j}-\frac{N^{2}}{\operatorname{Pr}_{h, v}}\right)^{1 / 2}\right],
$$

where $C_{s}$ is a constant, $N$ is the Brunt-Väisälä frequency, and $\operatorname{Pr}_{h, v}$ is the turbulent Prandtl number. The subscripts $h, v$ indicate different values for horizontal and vertical eddy viscosities, respectively. $C_{s}$ is typically 0.25 (default in WRF), but a value of $C_{s}=0.18$ has also been suggested in the literature (Sullivan et al. 1994) and is used in the LES test case distributed with WRF; both values will be considered here. The horizontal and vertical turbulence length scales, $l_{h}$ and $l_{v}$, can either be defined anisotropically as $l_{h}=\left(\Delta_{x} \Delta_{y}\right)^{1 / 2}, l_{v}=\Delta_{z}$ or isotropically as $l_{h}=l_{v}=\left(\Delta_{x} \Delta_{y} \Delta_{z}\right)^{1 / 3}$ (Skamarock et al. 2008). For consistency, anisotropic mixing lengths are used in all cases presented here, as recommended by the WRF guidelines when $\Delta_{x, y}$ and $\Delta_{z}$ are not of the same order. Isotropic mixing lengths were tested and found to have little effect for the finescale cases, where $\Delta_{x, y} \approx \Delta_{z}$, but had a noticeable detrimental effect for the coarse cases where the value of $K_{v}$ was increased along with $K_{h}$ despite $\Delta_{z}$ being unchanged. This will be described further in section 4 . The scalar eddy diffusivities are defined using $K_{h, v}^{\text {scalar }}=K_{h, v} / \operatorname{Pr}_{h, v}$, where $\operatorname{Pr}_{h}^{-1}=3$ and $\operatorname{Pr}_{v}^{-1}=1+2 l_{v} / \Delta_{z}$.

\section{b. The prognostic TKE (TKE-1.5) model}

The turbulent kinetic energy order-1.5 model (often called TKE-1.5) solves a prognostic TKE equation which includes TKE advection and parameterizes sources and sinks from shear production, buoyancy, turbulent mixing and dissipation (Skamarock et al. 2008). The TKE-1.5 closure uses a grid-based length scale to define the eddy-viscosity as

$$
K_{h, v}=C_{k} l_{h, v} e^{1 / 2},
$$

where $e$ is the TKE and $C_{k}$ is a constant, typically $0.15<C_{k}<0.25$. Similar to the Smagorinsky coefficient, the WRF default value is $C_{k}=0.15$, however the LES test case included in WRF's distribution specifies $C_{k}=0.10$; both values will be considered. The horizontal and vertical length scales, $l_{h}$ and $l_{v}$, are defined anisotropically as $l_{h}=\left(\Delta_{x} \Delta_{y}\right)^{1 / 2}$ and

$$
l_{v}= \begin{cases}\min \left[\Delta_{z}, 0.76 \frac{e^{1 / 2}}{N}\right] & : N^{2}>0 \\ \Delta_{z} & : N^{2} \leq 0\end{cases}
$$

in WRF, where the stability-based damping effect on the mixing length is similar to the damping applied in the Smagorinsky model. Scalar eddy diffusivities are defined for TKE-1.5 in the same way as the Smagorinsky model above.

\section{c. The dynamic reconstruction model (DRM)}

The dynamic reconstruction model considers both the explicit and implicit filters involved in LES. The DRM 
allows for backscatter of energy from small to large scales, exhibits excellent correlation with turbulent stresses obtained from a priori tests, and reduces numerical errors in the solution (Chow et al. 2005; Zhou and Chow 2011, 2012).

The LES filter is the operator represented by the overbar in Eq. (1). In most LES implementations, this filter is implicitly represented by the grid discretization and the discrete differentiation operation. Thus this implicit filter sets the effective minimum resolution, below which the cumulative effect of numerical truncation errors so strongly impairs motions that they are essentially absent. The DRM uses an explicit filter (larger than the grid cell width) combined with velocity reconstruction, previously described by Chow et al. (2005) and Gullbrand and Chow (2003). Specifically, explicit filtering separates the role of the LES filter from the implicit grid discretization effects. This means there is a range of eddy motions between the grid's Nyquist limit and the LES filter. These motions are, by definition, resolvable, because they are larger than the minimum resolvable wavelength on the grid, and are referred to as RSFS motions.

Using the explicit filter (overbar) and the implicit grid filter (denoted by a tilde), we define $\widetilde{u}_{i}$ as the resolvable velocity field on the grid, $\overline{\widetilde{u}_{i}}$ as the filtered, resolved velocity field and $\tilde{u}_{i}^{\star}$ as the reconstructed velocity field (Gullbrand and Chow 2003). The explicit filter is chosen here to be a tophat filter and is used in a deconvolution step to reconstruct the velocity field,

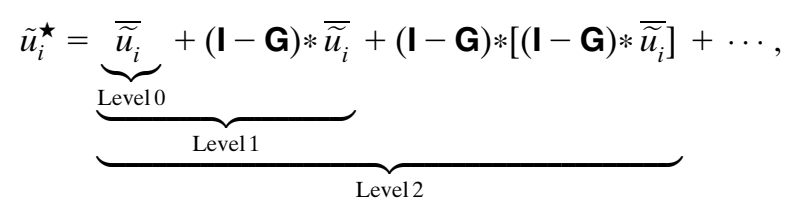

where $\mathbf{I}$ is the identity matrix, $\mathbf{G}$ is the explicit filter, and * represents the convolution operator (Gullbrand and Chow 2003). The DRM is characterized by the number of reconstruction terms included; level- $n$ reconstruction includes the first $n+1$ terms in the series [as indicated in Eq. (5)]. In practice, only the first few terms in the series are retained for $\tilde{u}_{i}^{\star}$ (Chow et al. 2005; Shi et al. 2018). Once $\tilde{u}_{i}^{\star}$ is reconstructed, the Wong and Lilly (1994) dynamic eddy-viscosity model (the Dynamic WongLilly model) is combined with the RSFS term to create a mixed model. Together these become

$$
\tau_{i j}=\left(\overline{\left(\tilde{u}_{i}^{\star} \tilde{u}_{j}^{\star}\right.}-\overline{\tilde{u}_{i}^{\star}} \overline{\tilde{u}_{j}^{\star}}\right)-2 c_{\varepsilon} \Delta_{f}^{4 / 3} \overline{\tilde{S}}_{i j},
$$

where $c_{\varepsilon}$ is a dynamically determined coefficient and $\Delta_{f}$ is the filter length scale (typically $2 \Delta_{x, y}$, which is used here). To prevent unstable, antidiffusive solutions, the dynamic coefficient of the eddy-viscosity term is constrained to $c_{\varepsilon} \Delta_{f}^{4 / 3}>-1.5 \times 10^{-5}$. Scalar diffusion uses a similar mixed model, where the scalar RSFS term is found with the same filtering process as for momentum [Eq. (5)] and the eddy-viscosity term is scaled by the same turbulent Prandtl number used with the Smagorinsky and TKE-1.5 models. For potential temperature $\theta$, the DRM SFS term is

$$
\tau_{\theta j}=\left(\overline{\tilde{\theta}^{\star} \tilde{u}_{j}^{\star}}-\overline{\tilde{\theta}^{\star}} \frac{1}{\tilde{u}_{j}^{\star}}\right)-\frac{1}{\operatorname{Pr}} 2 c_{\varepsilon} \Delta_{f}^{4 / 3} \frac{\partial \overline{\tilde{\theta}}}{\partial x_{j}} .
$$

Further details can be found in Chow et al. (2005).

When $\tilde{u}_{i}^{\star}=\overline{\tilde{u}}_{i}$ (level-0 DRM), we obtain a dynamic mixed model similar to Zang et al. (1993). We will consider the level-0 and level-2 DRM cases here, referred to as DRM0 and DRM2, respectively. Mixed models have long been shown to improve the performance of LES. Moeng et al. (2010), for example, conducted an a priori test on a cloud-resolving model with a horizontal resolution of $100 \mathrm{~m}$ and found that a mixed model greatly improved the representation of SGS fluxes of heat, moisture, and momentum.

\section{Case description}

This study considers the development of the CBL, a key aspect of NWP, in an idealized model setup featuring a quiescent initial state with no forced mean wind, a flat bottom boundary with uniform roughness, and periodic lateral boundary conditions. The simulations are run for $9 \mathrm{~h}$ with a constant sensible surface heat flux of $270 \mathrm{~W} \mathrm{~m}^{-2}$, the default value for idealized convective cases in WRFLES. This case was chosen primarily to isolate free convection, but also because it does not require any changes to the WRF code. The Wangara Day 33 case (Clarke et al. 1971) was also considered and yielded very similar results to those presented here (not shown).

A third-order Runge-Kutta time-integration scheme, a fifth-order horizontal advection scheme and a third-order vertical advection scheme are used (WRF default values). All cases use a time step of $0.1 \mathrm{~s}$, a domain height of $3.5 \mathrm{~km}$, and 65 vertical levels. The lowest vertical level is at $z=24 \mathrm{~m}$ and there are 10 levels below $z=500 \mathrm{~m}$. The surface drag is computed using the Monin-Obukhov surface layer scheme in WRF for all of the turbulence models used.

Simulations are performed with horizontal resolutions ranging from $\Delta_{x, y}=25 \mathrm{~m}$ to $1 \mathrm{~km}$ (see Table 1). Domains with $\Delta_{x, y} \leq 200 \mathrm{~m}$ are designed to have a size of $20 \mathrm{~km} \times 20 \mathrm{~km}$. The coarsest domains $\left(\Delta_{x, y}=500 \mathrm{~m}, 1 \mathrm{~km}\right)$ are larger in size to obtain adequate statistics with the lower 
TABLE 1. Domain sizes for each horizontal resolution considered for all turbulence models unless stated otherwise (as noted in sections $4 \mathrm{a}$ and $4 \mathrm{~d})$.

\begin{tabular}{rcc}
\hline \hline$\Delta_{x, y}(\mathrm{~m})$ & $n_{x, y}$ & Domain size $\left(\mathrm{km}^{2}\right)$ \\
\hline 25 & 800 & $20 \times 20$ \\
50 & 400 & $20 \times 20$ \\
100 & 200 & $20 \times 20$ \\
200 & 100 & $20 \times 20$ \\
500 & 80 & $40 \times 40$ \\
1000 & 80 & $80 \times 80$ \\
\hline
\end{tabular}

resolution (Table 1). Different domain sizes are used for some cases in sections $4 \mathrm{a}$ and $4 \mathrm{~d}$, as explained in the respective sections. Experiments were also conducted with finer and coarser vertical resolutions, but changes in vertical resolution had little to no impact on the results seen here. WRF version 3.8.1 is used for all cases.

The focus of this study is the grid dependence of largeeddy simulations, rather than the specific accuracy relative to field data. Therefore, the highest-resolution grid for a given turbulence model is considered to be the "true" solution for this idealized setup. A well-resolved solution for this convection-dominated case should show a shallow superadiabatic layer at the surface, a well-mixed neutral layer above and resolved entrainment of the free atmosphere at the top of the CBL (Stull 1988). The finest grid spacing should put the filter-width well within the inertial subrange, and the next-finest grid should show a nearly identical solution to confirm that our study begins in a region of gridindependent dynamics.

\section{Results}

\section{a. Mean CBL development}

The evolution of the CBL is first evaluated by considering hourly mean potential temperature profiles for the Smagorinsky, TKE-1.5, DRM0, and DRM2 models at the finest resolution $\left(\Delta_{x, y}=25 \mathrm{~m}\right)$. Smagorinsky and TKE-1.5 here use the WRF default coefficients where $C_{s}=0.25$ and $C_{k}=0.15$, respectively. Figure 1 shows that for all four models, the 25 -m cases show very good agreement in the development of the CBL. Specifically, they feature a well-mixed surface with a visible but shallow superadiabatic layer, a deepening neutral layer, and entrainment at the CBL top, as expected (Stull 1988). For all models, the 50-m cases (not shown) exhibit the same development and are nearly identical to the 25-m cases.

Now considering the $\Delta_{x, y}=1 \mathrm{~km}$ case for the Smagorinsky model using the WRF default value of $C_{s}=0.25$ (Fig. 2a), the mixing is clearly not sufficient to create the desired

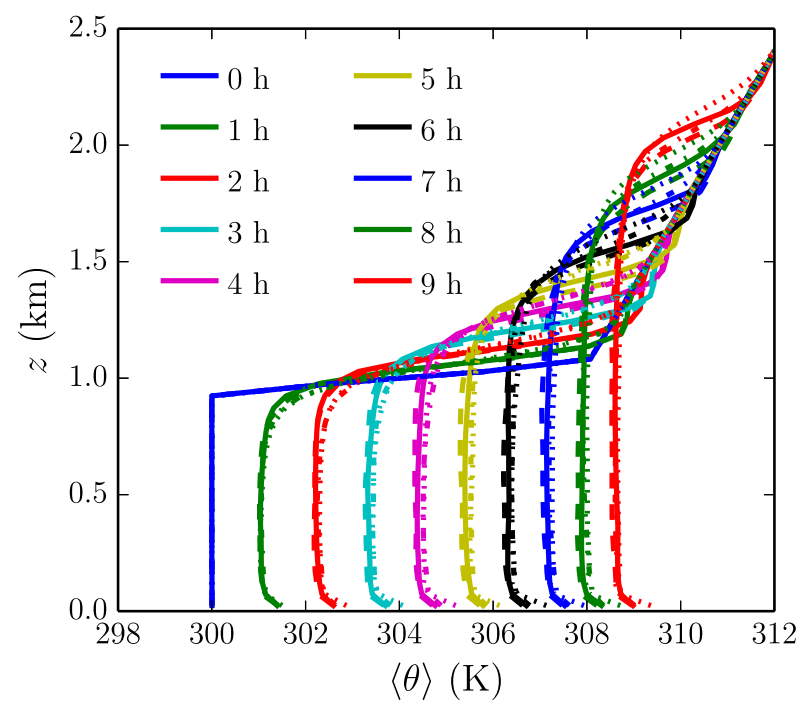

FIG. 1. Hourly, horizontally domain-averaged potential temperature profiles using the Smagorinsky (solid), TKE-1.5 (dashed), DRM0 (dash-dotted), and DRM2 (dotted) models for the 25-m resolution case.

neutral layer. This is especially true in the first four hours where the profile does not diverge from the initial profile above a height of approximately $900 \mathrm{~m}$. It should be noted that the potential temperature profiles for the Smagorinsky model in the WRF, presented here, may be sensitive to model parameters. For example, Efstathiou and Beare (2015) presented results using the Smagorinsky model in the Met Office Large Eddy Model, which uses different stability functions and a height-dependent length scale; their profiles, while still insufficiently mixed, appear better than the WRF results at coarse resolutions, especially near the surface.

Simulations using the Smagorinsky model that are coarser than $50-\mathrm{m}$ resolution are all associated with a deeper superadiabatic layer at the surface, even in the later hours when the profiles appear to be somewhat well mixed. The observed delay in resolved mixing for the Smagorinsky model can be seen more clearly by considering the evolution of the mean resolved vertical heat flux, $\left\langle w^{\prime} \theta^{\prime}\right\rangle$, in a horizontal plane $218 \mathrm{~m}$ above the surface (Fig. 3). The 25-, 50-, and 100-m cases show very similar behavior: resolved mixing begins approximately $15 \mathrm{~min}$ into the simulation, with the initially trapped warm air being released in a burst of buoyancy-induced mixing. In the 200-m case, a 25-min delay in the resolved heat flux onset is observed. Furthermore, the 500-m case is delayed a full hour, and the $1-\mathrm{km}$ case is delayed $2.5 \mathrm{~h}$. For all resolutions, the onset of resolved mixing is associated with a spike in $\left\langle w^{\prime} \theta^{\prime}\right\rangle$. For $\Delta_{x, y} \leq 200 \mathrm{~m},\left\langle w^{\prime} \theta^{\prime}\right\rangle$ reaches a quasi-steady value in the first hour, while the coarser resolutions take increasingly longer to reach a 

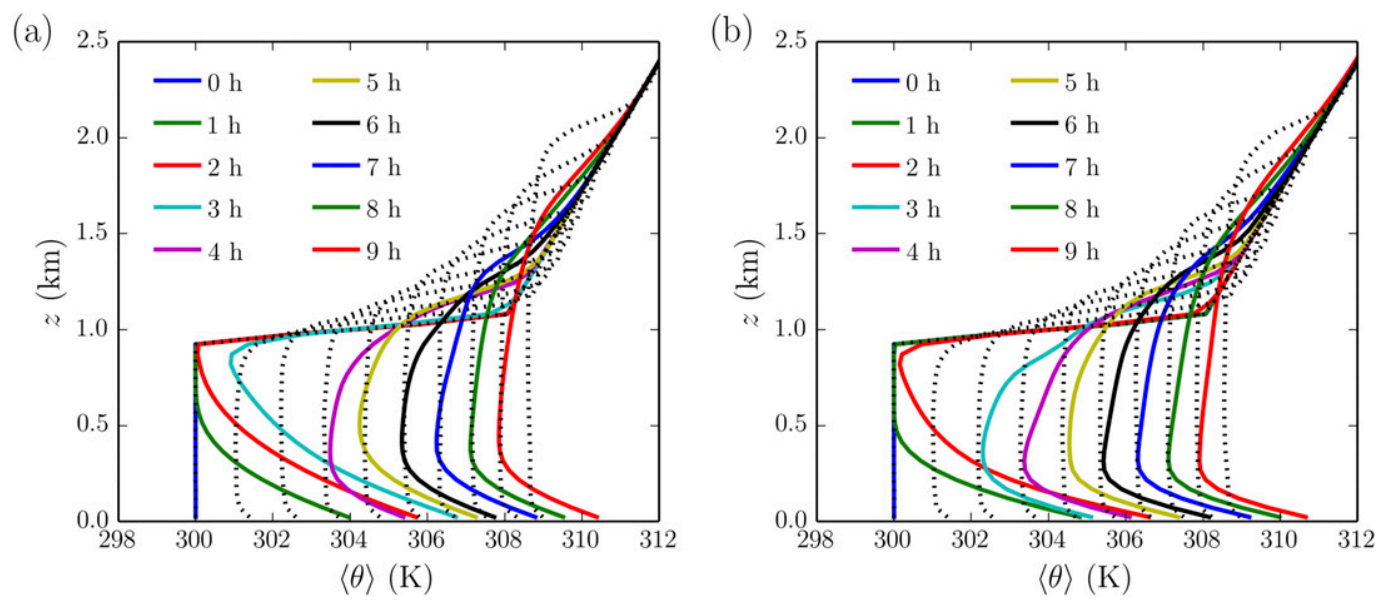

FIG. 2. Hourly, horizontally domain-averaged potential temperature profiles using the Smagorinsky model with (a) $C_{s}=0.25$ and (b) $C_{s}=0.18$ for $\Delta_{x, y}=1 \mathrm{~km}$, with profiles from the corresponding 25-m cases overlaid (dotted black).

steady mean planar value. Reconsidering the Smagorinsky model's mean potential temperature profiles with the knowledge of suppressed resolved turbulent mixing in the early hours of the simulation, we see that resolved mixing is very important to the potential temperature profiles. Before resolved turbulence begins, the surface heat flux is trapped in the lower atmosphere because the SFS turbulence model is only designed to provide local diffusion and is thus unable to mix the column when resolved mixing is absent. After resolved turbulence begins and the model is fully spun-up, the profiles change considerably in shape and it is clear that there is mixing throughout the column; however, these profiles are still very inaccurate in comparison with the higherresolution simulations.

The TKE-1.5 model, using the WRF default value $C_{k}=0.15$, appears to perform much better at coarser resolutions based on the potential temperature profiles, and even the 1-km case agrees relatively well with the 25-m case (Fig. 4a). There is a smaller delay in resolved vertical heat flux in the $218-\mathrm{m}$ plane, with the onset of the $1-\mathrm{km}$ case being delayed approximately $30 \mathrm{~min}$ and taking about $2 \mathrm{~h}$ to become steady (Fig. 5).

Using smaller coefficient values of $C_{s}=0.18$ and $C_{k}=0.10$ for the Smagorinsky and TKE-1.5 models, respectively, does show a small degree of improvement in potential temperature profiles for both models. The Smagorinsky model has slightly different results with the lower $C_{s}=0.18$ coefficient at coarser resolutions, where profiles show resolved mixing earlier (Fig. 2b). However, once both cases begin to resolve mixing, the $C_{s}=0.18$ profiles are less neutral than their $C_{s}=0.25$ counterparts, and both cases have a superadiabatic layer that is very sharp and extends more than $200 \mathrm{~m}$ vertically. For the lower $C_{s}$, the delay in resolved $\left\langle w^{\prime} \theta^{\prime}\right\rangle$ is reduced by about an hour in the $1-\mathrm{km}$ case and by $30 \mathrm{~min}$ in the 500-m case (not shown). The TKE-1.5 model, which already agrees relatively well with its 25 -m case at coarse resolutions, improves noticeably via sharper gradients in $\theta(z)$ resulting in shallower surface layers and sharper inversions (Fig. 4b). The TKE-1.5 model using the lower $C_{k}$ also sees the delay in resolved $\left\langle w^{\prime} \theta^{\prime}\right\rangle$ reduced to only $15 \mathrm{~min}$ when $\Delta_{x, y}=1 \mathrm{~km}$ and shows no delay in the 500-m case (not shown).

In an attempt to improve the performance of the Smagorinsky and TKE-1.5 cases, isotropic length scales $\left[l_{h}=l_{v}=\left(\Delta_{x} \Delta_{y} \Delta_{z}\right)^{1 / 3}\right]$ were also considered. Results are not presented here as all cases performed worse than their anisotropic counterparts. This is expected given the improvements seen using lower $C_{s, k}$ values, because isotropic length scales effectively only serve to increase $K_{v}$.

Examining the DRM, we see that for DRM0 the potential-temperature profiles show a well-mixed structure

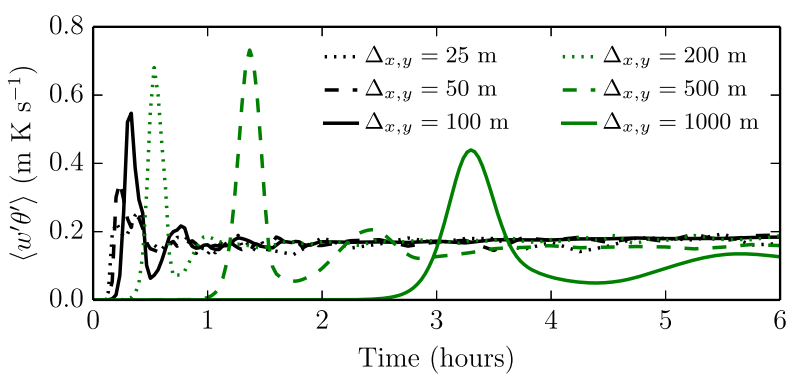

FIG. 3. Mean value of resolved $w^{\prime} \theta^{\prime}$ in the $218-\mathrm{m}$ plane (fifth vertical level) using the Smagorinsky model with $C_{s}=0.25$ for resolutions from $25 \mathrm{~m}$ to $1 \mathrm{~km}$. Planar averages are taken every $2 \mathrm{~min}$ initially and every $15 \mathrm{~min}$ once $\left\langle w^{\prime} \theta^{\prime}\right\rangle$ is approximately steady. 

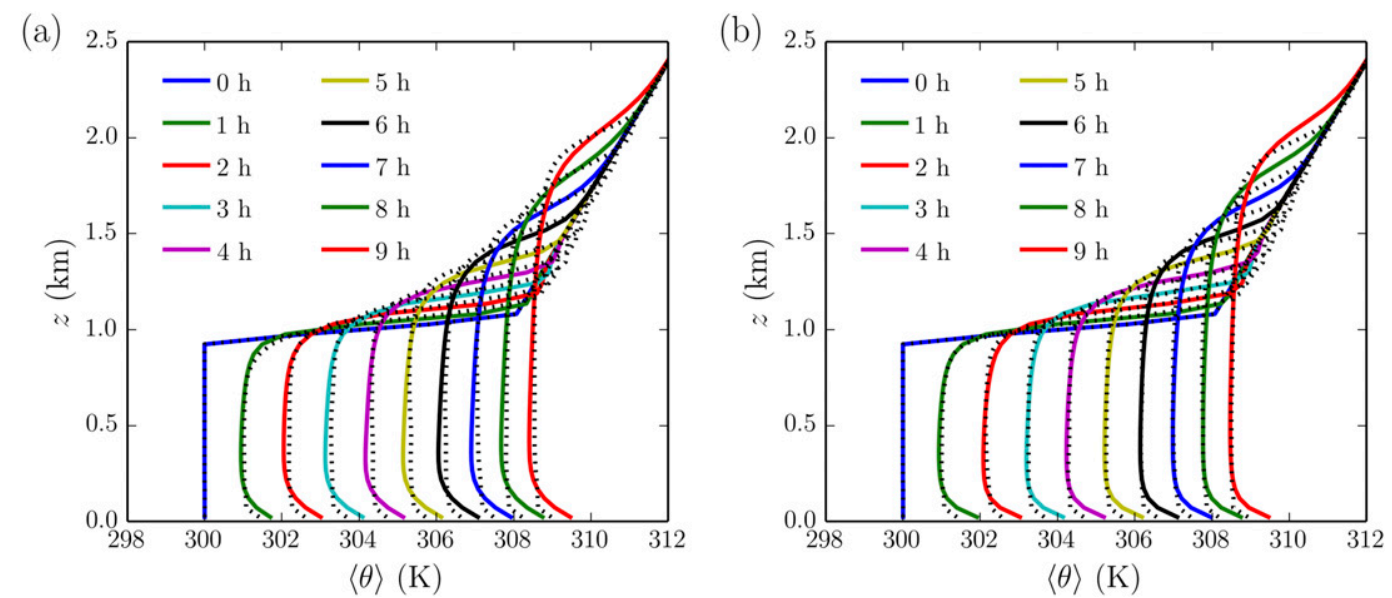

FIG. 4. As in Fig. 2, but for the TKE-1.5 model with (a) $C_{k}=0.15$ and (b) $C_{k}=0.10$.

throughout the simulation even at $1-\mathrm{km}$ resolution. The superadiabatic surface layer and inversion layer are much closer to the $25-\mathrm{m}$ case compared to equivalent resolutions using either Smagorinsky or TKE-1.5, even when using decreased values for $C_{s}$ and $C_{k}$ (Fig. 6a). Using the DRM0, the onset of resolved convection in the $218-\mathrm{m}$ plane shows a delay of less than $30 \mathrm{~min}$ for the $1-\mathrm{km}$ case, slightly better than the TKE-1.5 model (Fig. 7). The mean resolved heat flux in the 1-km case takes approximately two hours to become steady. The $500-\mathrm{m}$ and $1-\mathrm{km}$ cases show a similar burst of $\left\langle w^{\prime} \theta^{\prime}\right\rangle$ when resolved convection begins, though this is shorter lived than in the TKE-1.5 case and much shorter than the Smagorinsky case.

The $\Delta_{x, y}=1 \mathrm{~km}$ potential temperature profiles for the DRM2 case also show great agreement with the corresponding $\Delta_{x, y}=25 \mathrm{~m}$ case, producing the most accurate entrainment layer of any of the models considered (Fig. 6b). The two levels of additional reconstruction (from DRM0 to DRM2) do add a small kink to the potential-temperature profiles near the surface, but the overall performance is quite good considering the coarseness of the grid and the performance of the eddyviscosity models at the same resolution. The time series of $\left\langle w^{\prime} \theta^{\prime}\right\rangle$ for the DRM2 show the earliest resolved convection at all resolutions, the smallest bursts of upward resolved heat flux at all resolutions, and the earliest steadying at all resolutions (Fig. 8). The coarse DRM0 and DRM2 potential temperature profiles show a very slight warm bias in the bulk of the CBL when compared to their 25-m profiles. As shown in Zhou and Chow (2014), increasing levels of reconstruction in the DRM allows the model to resolve more turbulent fluxes under stably stratified environments. Compared to the DRM0 in Fig. 6a, the 1-km DRM2 predicts slightly larger boundary layer-top entrainment due to its higher reconstruction level, resulting in a warmer CBL. The performance the DRM under stably stratified conditions at kilometer-scale resolution will be examined in a future study.

Considering the time series of $\left\langle w^{\prime} \theta^{\prime}\right\rangle$ of the four models, it is expected that an LES will resolve less of any flux as the grid spacing becomes larger, because more energy is present in the SFS motions. The DRM0 and especially the DRM2 exhibit this behavior clearly while the Smagorinsky and TKE-1.5 models maintain a similar resolved vertical heat flux magnitude for all resolutions considered. This is due to gray-zone issues such as overly energetic resolved thermals and inaccurate SFS stress magnitudes in the latter two models.

The time series for $\left\langle w^{\prime} \theta^{\prime}\right\rangle$ when $\Delta_{x, y}=25$ and $50 \mathrm{~m}$ for all models are taken from smaller $80 \times 80$ domains with high temporal output to minimize data storage.

\section{b. Turbulent vertical momentum stress profiles}

Profiles of vertical turbulent momentum stresses $\left(w^{\prime} w^{\prime}\right)$ at $t=6 \mathrm{~h}$ for the $\Delta_{x, y}=500 \mathrm{~m}$ and $\Delta_{x, y}=1 \mathrm{~km}$ cases are compared to filtered high-resolution cases for further evaluation in Fig. 9. Vertical fluxes of horizontal momentum $\left(u^{\prime} w^{\prime}, v^{\prime} w^{\prime}\right)$ are not considered here because

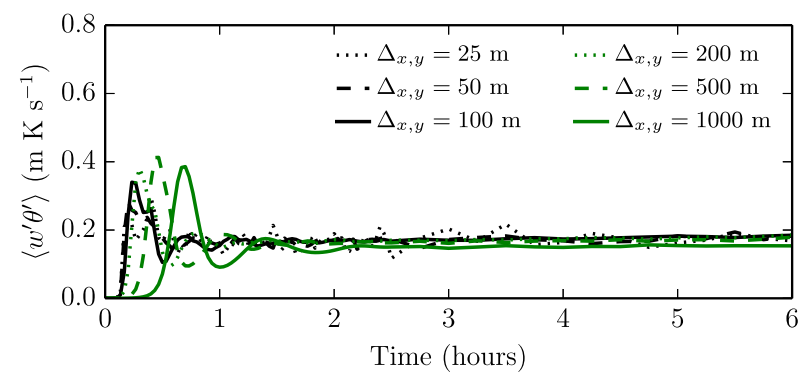

FIG. 5. As in Fig. 3, but for the TKE-1.5 model with $C_{k}=0.15$. 

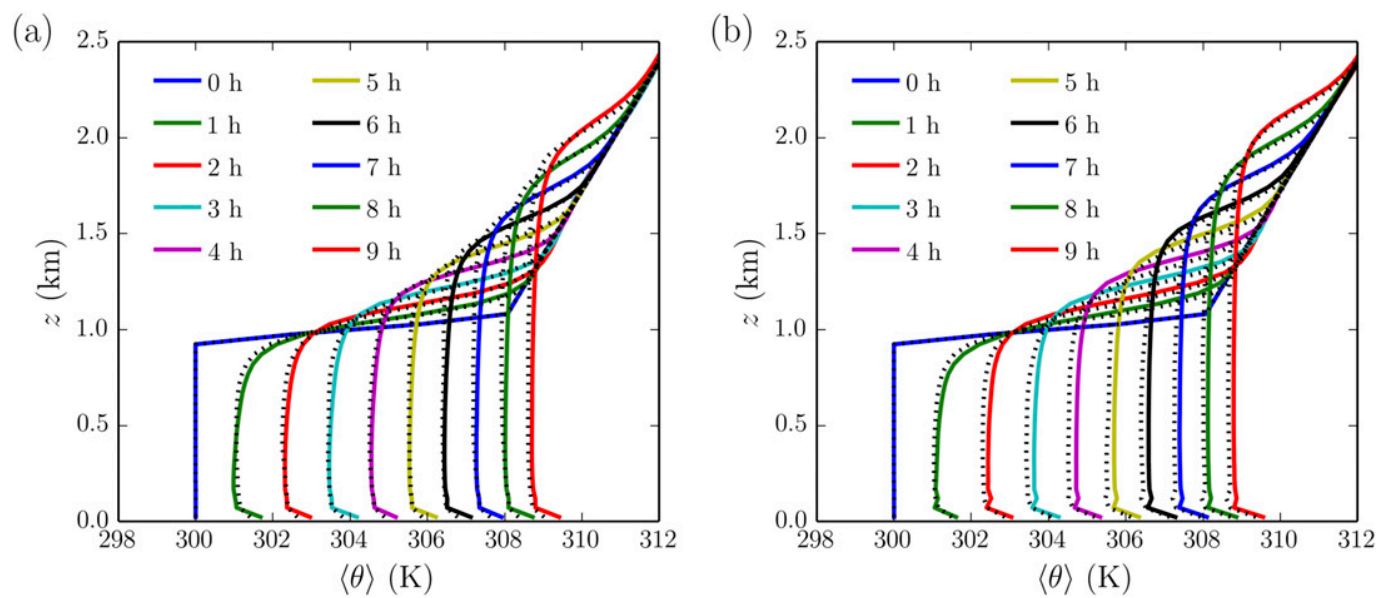

FIG. 6. As in Fig. 2, but for the (a) DRM0 and (b) DRM2.

there is no mean wind present. The total (resolved + SFS) stress profiles should be consistent for different grid resolutions for the LES to be considered accurate. The resolved and SFS profiles are also shown individually for the TKE-1.5, DRM0, and DRM2 models to further illustrate their behavior as the grid coarsens. The highresolution cases used for reference are the 25-m TKE-1.5 and DRM2 cases, which are filtered in each horizontal plane using a spectral cutoff filter where energy associated with a wavelength smaller than $2 \Delta_{\text {filter }}$ is set to zero. The TKE-1.5 and DRM2 high-resolution cases are sufficient for comparison as the resolved stress profiles for the 25-m TKE-1.5 and Smagorinsky cases are nearly indistinguishable, as are the total stress profiles for the 25-m DRM2 and DRM0 cases.

Only the resolved profiles are presented for the Smagorinsky model because it does not store the isotropic portion of $\bar{S}_{i i}$ and thus an accurate $\tau_{33}$ term cannot be found. The Smagorinsky model's resolved profile of $\left\langle w^{\prime} w^{\prime}\right\rangle$ is very similar to the DRM2 at $\Delta_{x, y}=500 \mathrm{~m}$, and is similarly shaped to the DRM2 but approximately half the magnitude at $\Delta_{x, y}=1 \mathrm{~km}$. It can be deduced from the resolved profiles and the model's poor overall performance in other metrics considered at coarse resolutions

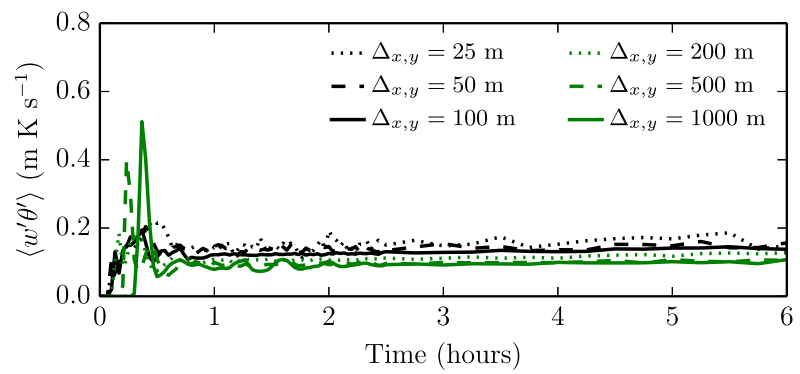

FIG. 7. As in Fig. 3, but for the DRM0. that the SFS contribution would not significantly improve its evaluation here. It will also be shown in later sections that the motions resolved by the Smagorinsky model occur at wavelengths that are much larger than those in the high-resolution case.

The planar-averaged SFS stress profiles for the TKE-1.5 model are found as $\left\langle\tau_{33}+2 e / 3\right\rangle$ to account for the isotropic portion of $\bar{S}_{i i}$. Considering the planaraveraged total vertical momentum fluxes shown in Fig. 9, the TKE-1.5 model performs relatively poorly for both the 500-m and 1-km cases when compared to the high-resolution cases. The TKE-1.5 model produces the largest planar-averaged resolved flux $\left(\left\langle w^{\prime} w^{\prime}\right\rangle\right)$ of all the models in the 500-m case and much more than any model except the DRM0 in the 1-km case. The 500-m TKE-1.5 case compares quite well to the filtered highresolution TKE-1.5 case, however, later analysis shows that these motions again occur at much larger wavelengths. The TKE-1.5 planar-averaged SFS stress $\left(\left\langle\tau_{33}\right\rangle\right)$ is the smallest of all of the models, however, generating a total flux profile that is much smaller in magnitude than either 25 -m case, especially when $\Delta_{x, y}=1 \mathrm{~km}$.

The SFS stress profiles for the DRM0 and DRM2 cases contain both the eddy-viscosity and the RSFS

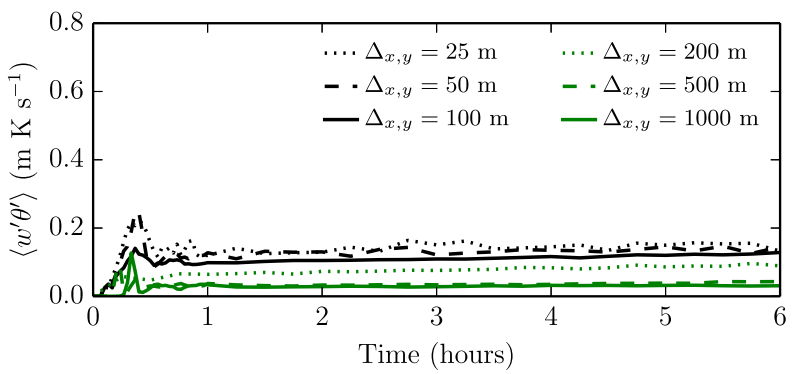

FIG. 8. As in Fig. 3, but for the DRM2. 

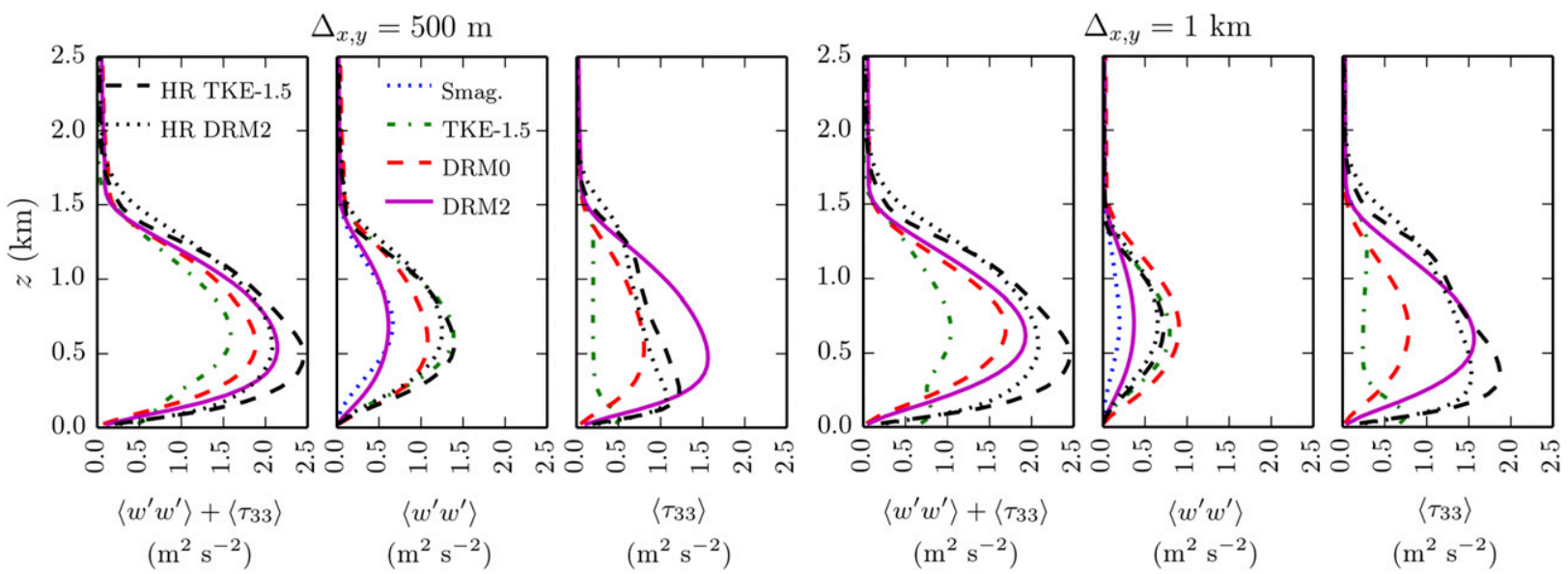

FIG. 9. Planar-averaged total (resolved + modeled) vertical momentum flux $\left(\left\langle w^{\prime} w^{\prime}+\tau_{33}\right\rangle\right)$, resolved vertical momentum flux $\left(\left\langle w^{\prime} w^{\prime}\right\rangle\right)$, and SFS vertical momentum flux $\left(\left\langle\tau_{33}\right\rangle\right)$ for the TKE-1.5, DRM0, DRM2, and Smagorinsky (resolved only) models after $6 \mathrm{~h}$ when (top) $\Delta_{x, y}=500 \mathrm{~m}$ and (bottom) $\Delta_{x, y}=1 \mathrm{~km}$, compared to high-resolution cases $\left(\Delta_{x, y}=25 \mathrm{~m}\right)$ using the TKE- 1.5 and DRM2 models filtered to the corresponding coarse resolution.

terms. The DRM0 has a much more significant SFS contribution than the TKE-1.5 model at both resolutions, leading to a total flux profile that is much closer to the TKE-1.5 and DRM2 high-resolution cases both in magnitude and shape, though still smaller than either high-resolution case in magnitude. The DRM2 has the largest SFS contribution of all the models due to the presence of the additional reconstruction terms representing the RSFS, resulting in the closest match to both high-resolution cases when considering the total flux profile. The DRM2 is especially accurate for the $\Delta_{x, y}=500 \mathrm{~m}$ case, where its total flux profile matches the DRM2 high-resolution case very closely.

Considering the total flux profiles, it is clear that the DRM2 is the closest match to the reference highresolution cases of all four models at both resolutions, followed closely by the DRM0. The TKE-1.5 model performs much better than the Smagorinsky model, but both perform poorly when compared to the DRM simulations.

Interestingly, filtering of the high-resolution TKE-1.5 case reveals a double-peak structure in the profile of $\tau_{33}$ that is much more exaggerated than for the DRM2, especially when filtered to $\Delta_{x, y}=1 \mathrm{~km}$. The DRM's ability to produce single-peaked second moments of resolved vertical velocity has been noted in other studies as well (Shi et al. 2018).

\section{c. Vertical velocity fields}

We next examine the vertical velocity field, $w(x, y)$, in the 218-m horizontal plane under coarsening horizontal resolution and compare to a high-resolution velocity field. The $218-\mathrm{m}$ plane is chosen for its visual clarity of the convective cells formed near the surface.
The coherent structures seen in $w$ indicate the size of the convective cells formed at each resolution, which can then be compared to high-resolution results. A spectral filter applied to a high-resolution case is then used to examine the coarse models' ability to accurately capture the proper scales of motion at that grid size.

At high resolutions all four models appear very similar and demonstrate the thermal cells that are expected for a convectively forced ABL with no mean wind. The TKE-1.5 model using 25-m horizontal resolution is shown in Fig. 10; the other three models are omitted because they are similar. As the horizontal resolution is coarsened, the size of the convective cells should not change and the resolved motions should be similar to those of the spectrally filtered high-resolution case.

Examining the resolved $w$ fields of the coarser cases using the Smagorinsky model $\left(C_{s}=0.25\right)$ shows the familiar cellular structure in the $100-\mathrm{m}$ case (Fig. 11). The 200-m case looks similar, but the cells are visibly larger in their characteristic size. This pattern continues for the 500-m and 1-km cases, where very large cells are resolved on the grid. Also note that these cells are much larger than the grid's Nyquist limit. Reducing the Smagorinsky coefficient to $C_{s}=0.18$ shows a reduction in the size of the thermal cells at coarser resolutions, though they remain extremely large compared to any of the high-resolution cases (Fig. 12). Note that all the $w$ slices are shown over a $20 \mathrm{~km} \times 20 \mathrm{~km}$ subdomain for comparison, though the actual simulations follow the domain sizes given in Table 1.

The resolved fields for the coarser cases using the TKE-1.5 model $\left(C_{k}=0.15\right)$ show a similar trend to the Smagorinsky model but to a smaller degree (Fig. 13). For comparison, the cells on the $1-\mathrm{km}$ grid using the 


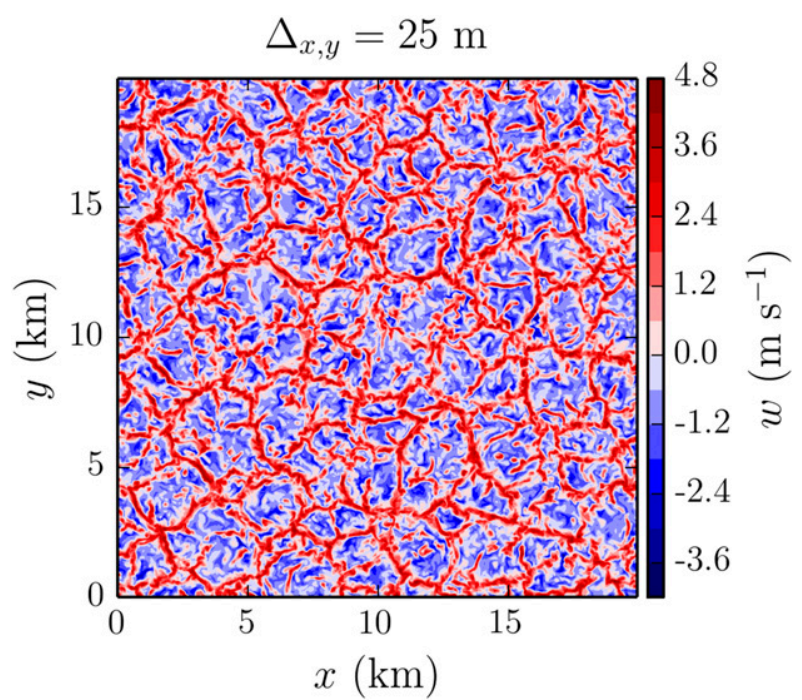

FIG. 10. Resolved vertical velocity $(w)$ field in the $218-\mathrm{m}$ plane at hour 6 for $\Delta_{x, y}=25 \mathrm{~m}$. All four models produced very similar fields; only the TKE-1.5 model is shown.

TKE-1.5 model appear similar in size to the cells seen on the 200-m grid using the Smagorinsky model, although the field is generally smoother between the updrafts of the thermal cells for the Smagorinsky model. Reducing the coefficient for the TKE-1.5 model to $C_{k}=0.10$ shows a similar reduction in the size of the thermal plumes, however the fields still contain very large structures (Fig. 14).

When using the DRM0 at fine resolutions $\left(\Delta_{x, y} \leq 200 \mathrm{~m}\right)$ the resolved vertical velocity field shows a cellular nature, as expected (Fig. 15). As the grid coarsens, the resolved $w$ field that the DRM0 produces becomes less coherent, in contrast to the large cells produced by standard eddy-viscosity models. The coarse DRM2 cases are qualitatively very similar to the DRM0 results, but with smaller magnitudes seen for both updrafts and downdrafts (Fig. 16). A quantitative comparison between the scales of energy present in the coarse cases and the scales present in the cellular, high-resolution cases is presented in section $4 \mathrm{~d}$.
For comparison to the coarser cases, the 25-m TKE-1.5 case is filtered in the same manner as in section $4 \mathrm{~b}$ (Fig. 17). The spectral filter selects the resolved largescale motions present in the high-resolution cases, which should ideally be reproduced by the coarse-resolution LES simulations. Filtering the high-resolution case using different filter cutoffs shows a resolved field which loses its cellular nature after a horizontal resolution of approximately $200 \mathrm{~m}$. The DRM0 and DRM2 are the only models which qualitatively agree with the filtered case at coarser resolutions, implying that the turbulent structures resolved by the two DRM models are more accurately represented than those from the eddy-viscosity models.

\section{d. Turbulent energy spectra}

The one-dimensional spectral energy densities of $w^{\prime}$ as a function of the horizontal wavenumber $k_{h}=\left(k_{x}^{2}+k_{y}^{2}\right)^{1 / 2}$ divided by the domain length $L$ for the Smagorinsky $\left(C_{s}=0.25\right)$, TKE-1.5 $\left(C_{k}=0.15\right)$, DRM0, and DRM2 models taken at $z=667 \mathrm{~m}$ (approximately the center of the CBL) after $6 \mathrm{~h}$ are shown in Fig. 18. All models agree very closely with each other as well as with the theoretical $k^{-5 / 3}$ decay rate at high resolutions $\left(\Delta_{x, y}=25 \mathrm{~m}\right.$ shown $)$, but disagree significantly when $\Delta_{x, y}=1 \mathrm{~km}$. The ideal spectrum at a coarse resolution would follow the highresolution spectra until the grid's Nyquist limit (Skamarock 2004). The Smagorinsky model is so dissipative that very little energy is resolved other than at very coarse modes, resulting in too much energy at the grid's coarse scales and not enough energy at the grid's fine scales, as was seen qualitatively in section $4 \mathrm{c}$. The TKE-1.5 model performs much better in that it allows much more energy to develop on the grid's finer scales. However, similar to Smagorinsky, the TKE-1.5 model allows too much energy to build up at coarser resolutions, generating the larger, cohesive structures seen in the $w(x, y)$ fields. This can be addressed to some degree via changing the $C_{s, k}$ values for the Smagorinsky and TKE-1.5 models, respectively (spectra not shown), which reduces the energy in long waves and increases the energy
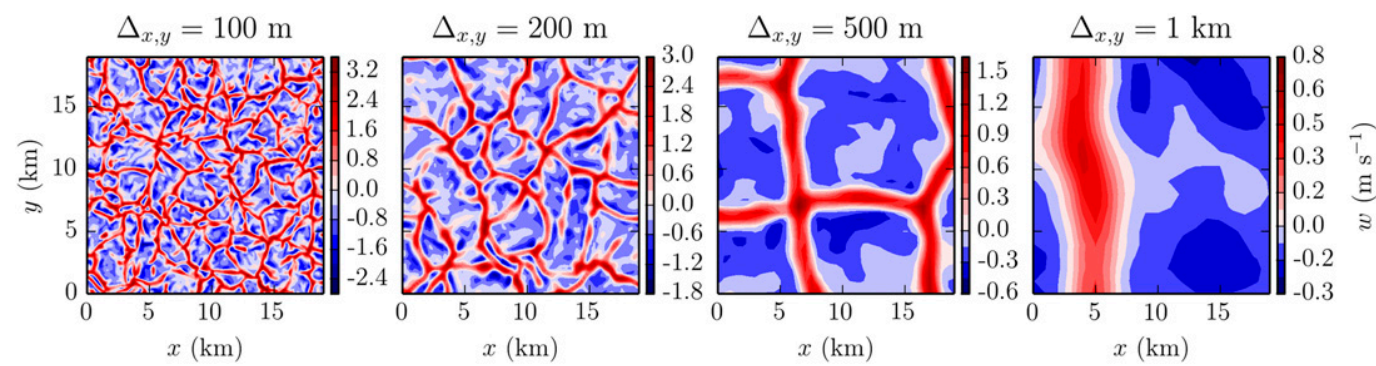

FIG. 11. Resolved vertical velocity $(w)$ field in the 218 -m plane at hour 6 using the Smagorinsky $\left(C_{s}=0.25\right)$ model for $\Delta_{x, y}=100,200,500$, and $1000 \mathrm{~m}$. All cases are plotted as $20 \times 20 \mathrm{~km}^{2}$, but actual domain sizes follow Table 1 . 

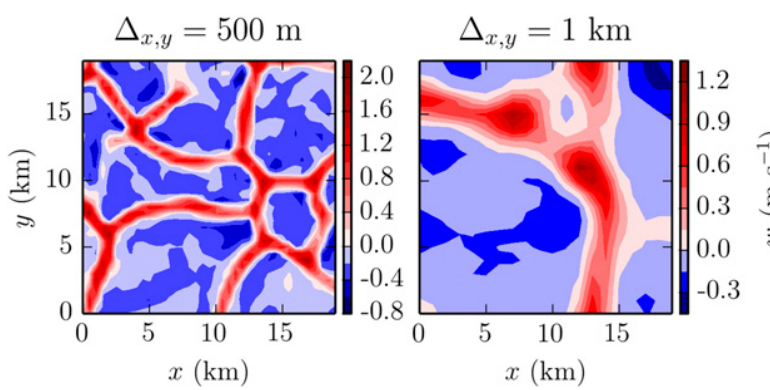

FIG. 12. As in Fig. 11, but for the Smagorinsky $\left(C_{s}=0.18\right)$ model for $\Delta_{x, y}=500 \mathrm{~m}$ and $1 \mathrm{~km}$.

in short waves, though this creates a very case-specific turbulence model that is still not particularly accurate. The DRM0 is the closest of the four models to matching the spectral density of the high-resolution cases, and excels both in terms of avoiding a buildup of energy at large scales and in allowing energy to develop on the grid's finer scales. The DRM0 also has the sharpest decay when approaching the grid's Nyquist limit, with a peak energy that is the closest of the four to the high-resolution cases.

It was seen in section $4 \mathrm{~b}$ that the DRM0 resolves more turbulent stress than the DRM2 due to the expected increase in the SFS term of the DRM2 from its RSFS terms. This relationship is seen again in the spectra, where the DRM0 and DRM2 agree closely at large scales but the DRM0 has more energy than the DRM2 at finer scales. This again is attributed to the DRM2 RSFS contribution, which is larger due to the higher level of velocity reconstruction (Chow et al. 2005). In section $4 \mathrm{~b}$, the TKE-1.5 and DRM0 models had very similar profiles of resolved variance, $\left\langle w^{\prime} w^{\prime}\right\rangle$, but we can see in the spectra (and in section 4c) that the TKE-1.5 model's resolved energy occurs at much larger wavelengths than the DRM0 despite their similar total resolved stress throughout the profile. This is consistent across energy spectra taken at different vertical levels (not shown).

To make a quantitative evaluation of the size of the thermal cells, the dominant wavelength of the resolved vertical velocity field is approximated by the critical wavelength $\lambda_{c}=1 / k_{c}$ (the $2 \pi$ is omitted because we are interested in meters per cycle, rather than meters per radian, to get a better physical intuition of the quantities); $k_{c}$ is defined as the wavenumber where $2 / 3$ of the velocity variance seen in the physical field $\left(\left\langle w^{\prime} w^{\prime}\right\rangle\right.$ in this case) is contained between $k_{c}$ and $k_{N}$, the Nyquist limit, on the energy spectrum $S$ of the turbulent velocity. For $w^{\prime}$ using a discrete Fourier transform, we can say

$$
\frac{2}{3}\left\langle w^{\prime} w^{\prime}\right\rangle=\sum_{k_{c}}^{k_{N}} S_{33}
$$

Values for $\lambda_{c}$ are found here by considering the same one-dimensional energy spectra shown in Fig. 18 and finding the smallest $k_{c}$ value where $2 / 3\left\langle w^{\prime} w^{\prime}\right\rangle \geq \sum_{k_{c}}^{k_{N}} S_{33}$ for each case. This can be considered similar to taking the median of a sample, and is used for its reduced sensitivity to noise in determining the peak of the spectrum (de Roode et al. 2004). The critical wavelength is computed in the same $218-\mathrm{m}$ plane seen in Figs. 10-17. Calculating $\lambda_{c}$ is sensitive to domain size and requires a domain large enough to have negligible energy at the largest scales. To this end, some cases required domains up to $25 \%$ larger in the horizontal to achieve statistics independent of domain size for $\lambda_{c}$.

In Fig. 19, all four models have a consistent dominant wavelength $\lambda_{c} \approx 1 \mathrm{~km}$ for $\Delta_{x, y} \leq 100 \mathrm{~m}$, and agree very well with each other. At $200 \mathrm{~m}$, the Smagorinsky, TKE-1.5 and DRM2 models begin to show increasing dominant wavelengths and all four models begin to diverge from each other. When $\Delta_{x, y}=500 \mathrm{~m}$ the Smagorinsky model's critical wavelength is greater than $6 \mathrm{~km}$ and by $\Delta_{x, y}=1 \mathrm{~km}$ the Smagorinsky model's critical wavelength has increased 20 -fold from the $\Delta_{x, y}=25 \mathrm{~m}$ case value of $\lambda_{c}=1.0 \mathrm{~km}$ to $\lambda_{c}=19.8 \mathrm{~km}$. The TKE-1.5 model's increase from $\lambda_{c}=1.1$ to $\lambda_{c}=4.6 \mathrm{~km}$ for the same change in resolution appears modest when compared to Smagorinsky, but it is still nearly a fivefold increase and is also more than twice the size of the grid's Nyquist limit of $2 \mathrm{~km}$.
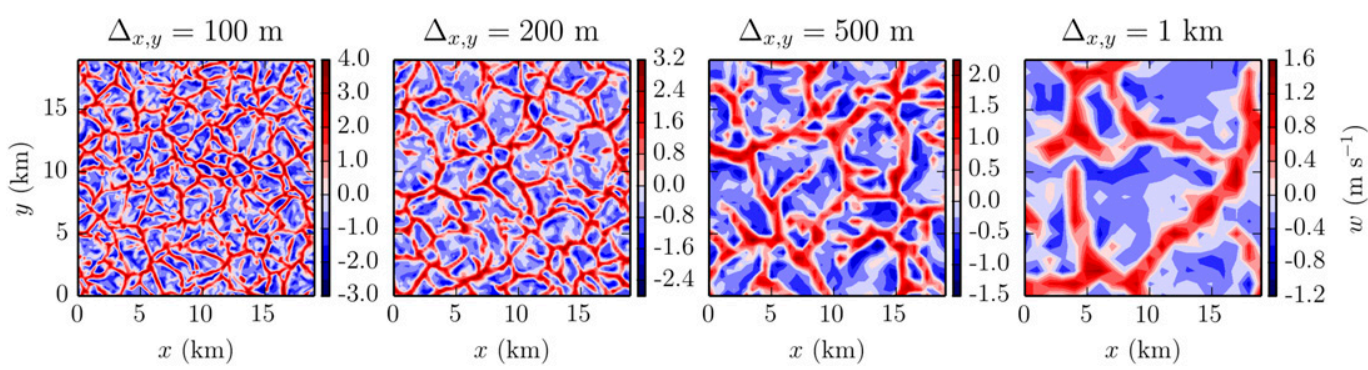

FIG. 13. As in Fig. 11, but for the TKE-1.5 $\left(C_{k}=0.15\right)$ model. 

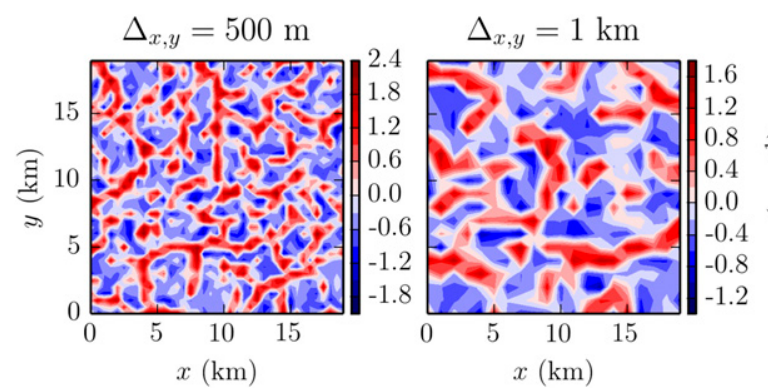

FIG. 14. As in Fig. 11, but for the TKE-1.5 $\left(C_{k}=0.10\right)$ model for $\Delta_{x, y}=500 \mathrm{~m}$ and $1 \mathrm{~km}$.

The DRM0 and DRM2 show a more consistent value for $\lambda_{c}$ as horizontal resolution increases. The DRM2, as noted above, shows a small increase when $\Delta_{x, y}=200 \mathrm{~m}$, but does not grow as quickly as TKE-1.5. Both DRM0 and DRM2 begin to show a notable increase in $\lambda_{c}$ when $\Delta_{x, y}=1 \mathrm{~km}$ with $\lambda_{c}=2.9 \mathrm{~km}$ for DRM0 and $\lambda_{c}=3.3 \mathrm{~km}$ for DRM2. Note that some increase is inevitable because the $\Delta_{x, y}=25 \mathrm{~m}$ critical wavelength $(\sim 1 \mathrm{~km})$ is smaller than the 1-km grid's Nyquist limit of $2 \mathrm{~km}$. Interestingly, the DRM2 has a slightly larger critical wavelength than the DRM0 at coarse resolutions and is somewhat comparable to the TKE-1.5 model by the $\lambda_{c}$ metric, especially at $\Delta_{x, y}=500 \mathrm{~m}$, however the magnitude of the resolved energy is much lower than the TKE1.5 model (seen in Figs. 9 and 18), resulting in a more accurate $w$ field.

As we have seen, different LES turbulence models have varying responses to coarse (gray zone) resolutions due to differences in their formulation, implying that the models should not necessarily share criteria for being in the gray zone. Along these lines, Beare (2014) explored the length scales of the gray zone and suggested that the conventional wisdom that the gray zone begins when $z_{i} \sim \Delta_{x, y}$ (where $z_{i}$ is the inversion depth) does not sufficiently capture the nuances of the turbulence model. They instead introduced the dissipation length scale $\lambda_{d}$, which is the wavelength where turbulent dissipation begins in the energy spectrum. The result of the horizontal resolution and underlying numerical schemes on a turbulence model's performance can be encompassed by $\lambda_{d}$, defined as $\lambda_{d}=1 / k_{d}$, where

$$
k_{d}^{2}=\frac{\sum k^{2} S_{33}}{\sum S_{33}} .
$$

Beare (2014) found that the gray zone begins when $\lambda_{p}=1.4 \lambda_{d}$, where $\lambda_{p}$ is the peak wavelength of the turbulent energy spectrum [here we are assuming $\lambda_{p} \approx \lambda_{c}$ from Eq. (8)]. When $\lambda_{c}$ is considered as a function of $\lambda_{d}$, instead of a function of $\Delta_{x, y}$, the relationships for the four models essentially overlap, indicating that the dissipation length scale is a much more general predictor of the gray zone than horizontal resolution (Fig. 20). After considering the energy spectra and $\lambda_{c}$ versus $\Delta_{x, y}$ for the four models, it is not surprising that the DRM0 and DRM2 also show the slowest increase in dissipation length scale as resolution coarsens.

Generally we agree with Beare (2014) that $\lambda_{c}$ or $\lambda_{d}$ are useful metrics for diagnosing the boundaries of the gray zone. Specifically, using $\lambda_{d}$ as the characteristic length scale for a given grid resolution, in lieu of $\Delta_{x, y}$, provides a much more accurate measure of the limits of motions that can be represented numerically. The dissipation length scale is also useful for comparing the gray zones for different turbulence models because it includes the effects of the specific models on the energy spectra, which is crucial to turbulent dynamics in the gray zone.

\section{e. Backscatter in the DRM}

Energy transfer between resolved and SFS scales is represented by the dissipation $\Pi$, defined as

$$
\Pi=-\tau_{i j} \bar{S}_{i j}
$$

When $\Pi$ is positive it represents dissipation (i.e., energy transfer from resolved to SFS scales, which is a primary function of an LES turbulence model). $\Pi$ is called dissipation because it is largely positive, although negative values (backscatter of energy from the SFS to the resolved
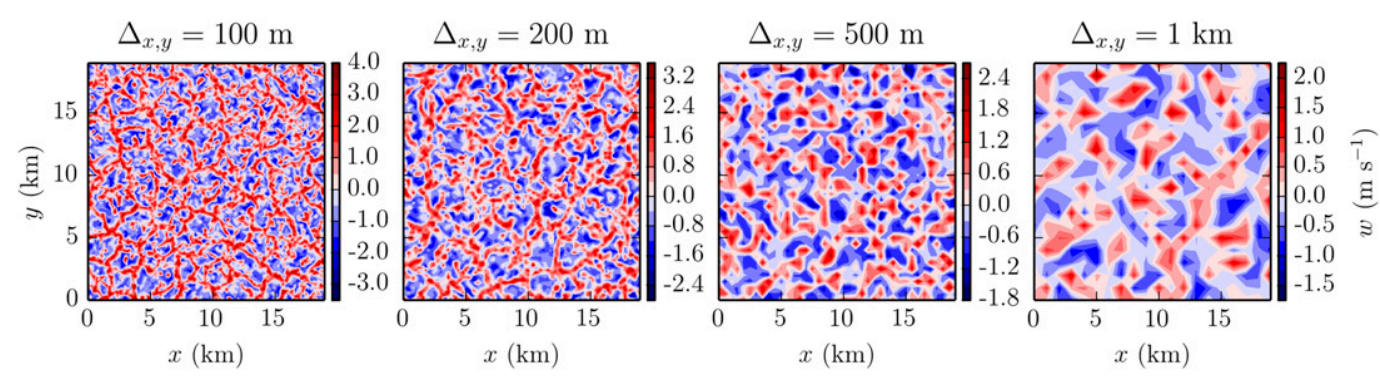

FIG. 15. As in Fig. 11, but for the DRM0. 



FIG. 16. As in Fig. 11, but for the DRM2.

scales) are also seen in atmospheric observations (PortéAgel et al. 2001; Carper and Porté-Agel 2004). Eddyviscosity models are purely dissipative by their formulation, which does not allow $K_{h, v}$ to take negative values. Because $\tau_{i j}$ and $\bar{S}_{i j}$ are always opposite in sign (or zero) for these models due to their definition of $\tau_{i j}$ [Eq. (2)], $\Pi$ can never be negative. Scale-similarity models are not constrained in the same way, and do allow for energy transfer from SFS to resolved scales via negative dissipation values (Zhou and Chow 2011).

PDFs of $\Pi$ for all four models considered, normalized by their standard deviations, $\sigma$, are shown for the $\Delta_{x, y}=200 \mathrm{~m}$ (Fig. 21a) and $\Delta_{x, y}=1 \mathrm{~km}$ (Fig. 21b) cases. Data from the fourth to twentieth vertical levels (from $z=218$ to $z=976 \mathrm{~m}$ ) are used to compute the PDF of dissipation values within the well-mixed layer of the boundary layer. PDFs at other grid resolutions show similar behavior and are not shown here. The Smagorinsky and TKE-1.5 models have only positive dissipation values, as expected. The DRM0 and DRM2 show a significant backscatter component and compare well to the observations of $\tau_{33}$ seen by Carper and Porté-Agel (2004). For the $\Delta_{x, y}=200 \mathrm{~m}\left(\Delta_{x, y}=1 \mathrm{~km}\right)$ case, $42 \%$ (47\%) of the grid points produce backscatter accounting for $35 \%$ (47\%) of the total stress by magnitude-weighted average using the DRM0, and $43 \%(52 \%)$ of the grid points produce backscatter accounting for $41 \%(56 \%)$ of the total stress by magnitude-weighted average using the DRM2. Backscatter in the DRM is nearly entirely attributed to the scale-similarity term. The eddy-viscosity component of the DRM is not a significant contributor to backscatter because the dynamic coefficient is constrained to $c_{\varepsilon} \Delta_{f}^{4 / 3}>-1.5 \times 10^{-5}$.

That the DRM creates backscatter is one reason that it performs well at coarse resolutions. Specifically, the DRM allows backscatter of energy from the SFS to the resolved scales, introducing resolved energy near the grid's Nyquist limit that is not seen in the eddy-viscosity models. The positive effects of backscatter are especially pronounced at the coarse resolutions considered here due to the important role of energy near the grid's Nyquist limit.

\section{f. Other considerations}

Given the sensitivity of the CBL evolution to the closure models, changes to several other aspects of the numerical configuration were also explored. Changing from third- to fifth-order advection has little influence on the dynamics (not shown). Changing the vertical advection to an even, fourth-order scheme, which does not contain any numerical diffusion, does tend to reduce the delay of resolved mixing by a few minutes in both the 500-m and 1-km cases (not shown). While the eddy-viscosity model is primarily responsible for the grid-dependent effects seen, the numerical diffusion present in the default third-order vertical advection term exacerbates the issue of overly damping the high wavenumbers. Changing the horizontal advection scheme between odd and even ordered does not have a noticeable effect on the dynamics for the convectively driven case considered here.

Increasing the vertical resolution has little effect on the size of the resolved thermal cells or the delay in resolved turbulence. Coarser vertical-resolution cases were also tested, in accordance with Mirocha et al. (2010), who advise an aspect ratio $\left(\alpha=\Delta_{x} / \Delta_{z}\right)$ of 2-4. Ultimately, even large changes in vertical resolution have little influence on the presence of large resolved thermal structures seen with coarse horizontal resolution. (Zhou et al. (2018) explored the use of increased

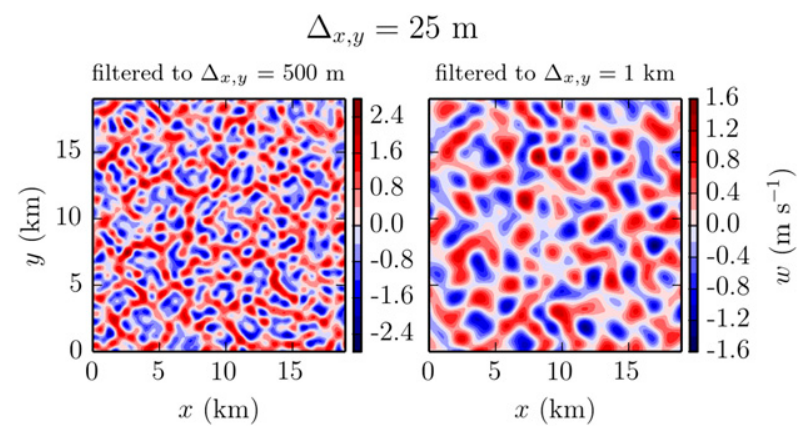

FIG. 17. Filtered resolved vertical velocity $(w)$ field in the 218-m plane at hour 6 for the 25-m TKE-1.5 case, filtered to (left) $\Delta_{x, y}=500 \mathrm{~m}$ and (right) $\Delta_{x, y}=1 \mathrm{~km}$. 


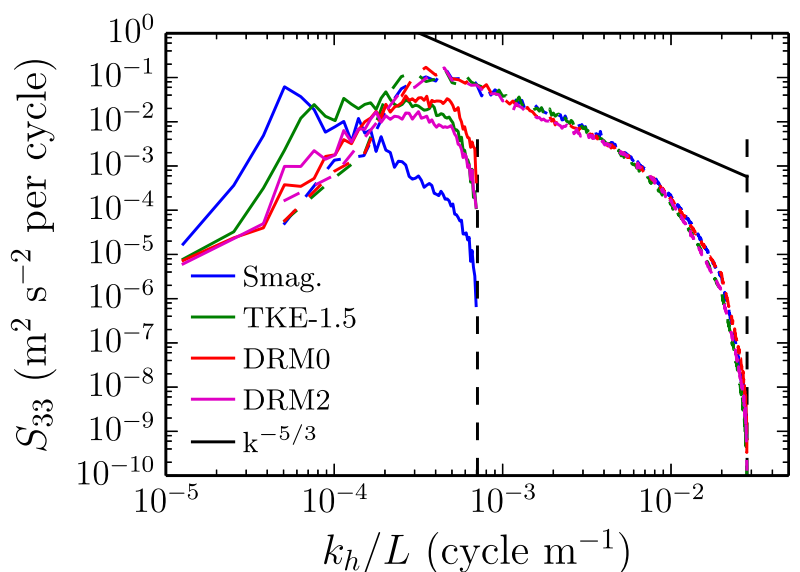

FIG. 18. Spectral energy density of $w^{\prime}$ as a function of $k_{h} / L$, where $k_{h}$ is the horizontal wavenumber and $L$ is the domain length, for the Smagorinsky, TKE-1.5, DRM0, and DRM2 models in the 667-m plane after $6 \mathrm{~h}$ at $25-\mathrm{m}$ (dashed) and 1-km (solid) resolutions. Nyquist limits of the respective grids also shown (dashed black).

horizontal refinement near the surface to alleviate the impact of unrealistic large thermal structures.)

Alternative surface-stress implementations [as in Mirocha and Lundquist (2017)] were used for the Smagorinsky model but had minimal impact on its performance and are not shown here. The Dynamic Wong-Lilly eddy-viscosity model alone, without any scale-similarity or velocity reconstruction, outperforms the Smagorinsky and TKE-1.5 models based on all of the criteria considered here, however it is inferior to the DRM0 and DRM2 and is thus not presented.

The current DRM implementation in WRF has not been optimized and requires approximately twice as long to run compared to the eddy-viscosity closure models. In other codes, the DRM has been found to increase computational cost by only $25 \%-60 \%$ depending on the level of reconstruction (Chow et al. 2005; Kirkil et al. 2012; Shi et al. 2018).

\section{Summary and conclusions}

The gray zone refers to a range of resolutions where there exists no fundamentally appropriate turbulence model. The gray zone for a CBL simulation includes resolutions that lie between the traditional application of mesoscale PBL parameterizations and LES closure models, and is often generally defined to be the range when horizontal resolutions are $\mathscr{Q}(1) \mathrm{km}$. The gray zone is specific not only to the mesoscale and finescale turbulence models chosen, but also to the physics of the case being simulated. Here, simulations performed at various resolutions and with different closure models allowed a detailed examination of the gray zone for the convectively forced atmospheric

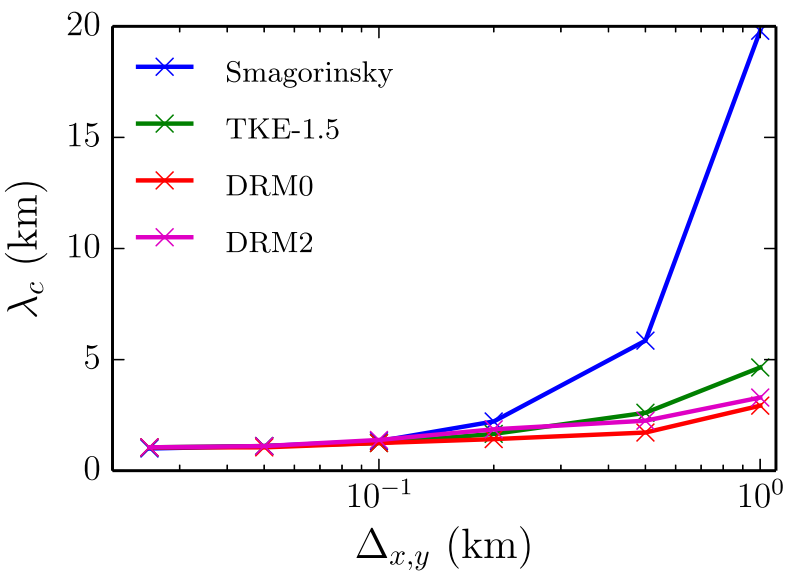

FIG. 19. Horizontal resolution vs critical wavelength of resolved $w^{\prime}$ in the 218-m plane after $6 \mathrm{~h}$ for Smagorinsky, TKE-1.5, DRM0, and DRM2 for resolutions from $25 \mathrm{~m}$ to $1 \mathrm{~km}$. Calculated by applying Eq. (8).

boundary layer. Vertical profiles of potential temperature and turbulent fluxes, time series of resolved fluxes, horizontal slices of vertical velocity fields and energy spectra of coarse simulations were compared to higherresolution simulations.

Based on the dependence of the critical wavelength $\lambda_{c}$ on horizontal resolution, the gray zone appears to begin at $\Delta_{x, y} \approx 200 \mathrm{~m}$ for both the Smagorinsky and TKE-1.5 models. This is in agreement with prior studies in the literature (Beare 2014; Zhou et al. 2014; Efstathiou and Beare 2015), which also include other numerical weather prediction models in addition to WRF. Both eddy-viscosity models are problematic at coarse resolutions because the finescale motions, relative to the grid, are damped, forcing the model to resolve turbulence at

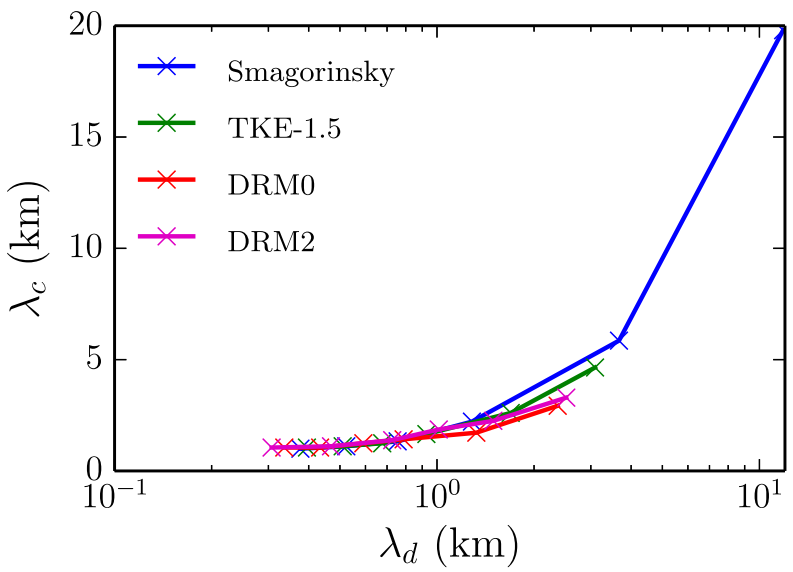

FIG. 20. Dissipation wavelength vs critical wavelength of resolved $w^{\prime} w^{\prime}$ in the 218-m plane after $6 \mathrm{~h}$ for Smagorinsky, TKE-1.5, DRM0, and DRM2 for resolutions from $25 \mathrm{~m}$ to $1 \mathrm{~km}$. Calculated by applying Eqs. (8) and (9). 

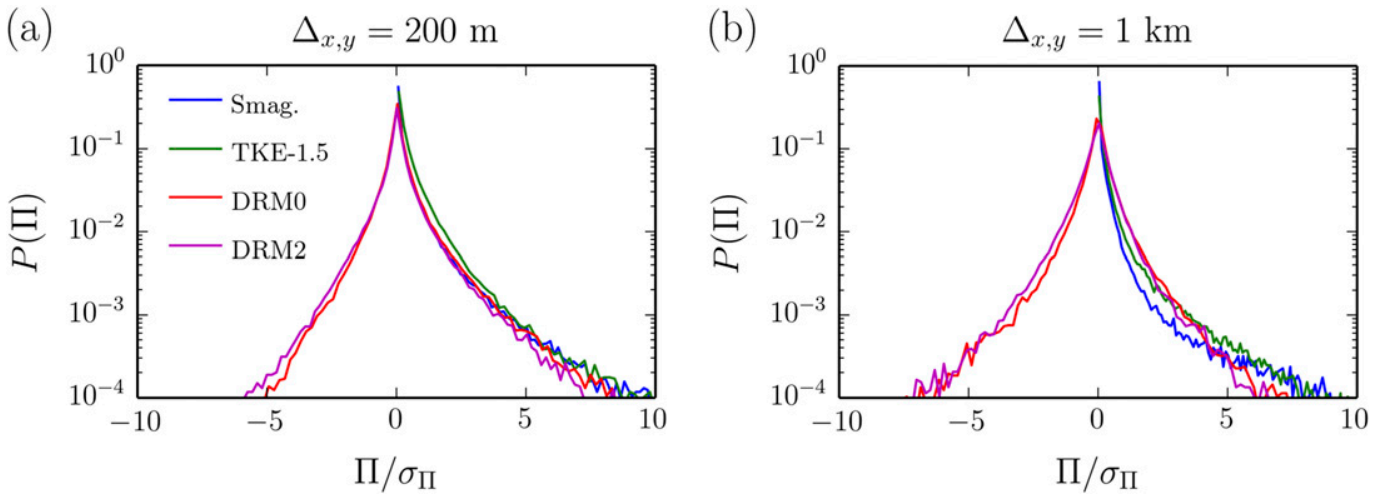

FIG. 21. PDF of dissipation in 16 horizontal planes, from the fourth to twentieth vertical level, at hour 6 for the Smagorinsky, TKE-1.5, DRM0, and DRM2 models at (left) 200-m and (right) 1-km (right) resolutions. The Smagorinsky and TKE-1.5 models have only positive dissipation values while the DRM0 and DRM2 both show a large backscatter component.

artificially large scales. The eddy-viscosity models also are unable to produce accurate total (resolved + SFS) stress profiles when compared to high-resolution LES statistics. However, the TKE-1.5 model, despite its deficiencies, is significantly superior to the Smagorinsky model in the gray zone.

The DRM is a mixed model which allows backscatter of energy from small to large scales. In the DRM the scale-similarity component is designed to reconstruct structures that are large enough to be resolved on the grid but are removed due to the implicit filter of the LES. Because the filter width is explicitly included in the DRM, it provides a potential solution for turbulence modeling at both LES resolutions and in the gray zone. Because of the DRM's explicit filtering, numerical errors are overall reduced (see Gullbrand and Chow (2003)). Then, reconstruction allows better representation of grid-scale turbulent motions (near the grid cutoff). Other dynamic model implementations have considered the choice of the test filter relative to the grid cutoff as a way to improve this further (SchaeferRolffs 2017). For example, using either the DRM0 or DRM2 at resolutions coarser than $200 \mathrm{~m}$ shows a much better qualitative and quantitative agreement with the high-resolution simulation results than the standard Smagorinsky or TKE-1.5 closures. The DRM2 especially excels at producing accurate total stress profiles at coarse resolutions when compared to high-resolution cases. By using the DRM turbulence closure, the extent of the gray zone is greatly reduced for this convective case. Explicit filtering and reconstruction using the DRM produces realistic dynamics using a horizontal resolution as coarse as $1 \mathrm{~km}$ without any tuning to account for either the coarseness of the grid or the physics of the case being considered. The ability of the DRM to produce realistic turbulence on a coarse grid by reconstructing
RSFS motions is very encouraging and will be the subject of future work where more complex cases will be considered.

Acknowledgments. We are grateful for support from a Presidential Early Career Award for Scientists and Engineers (PECASE) and from National Science Foundation (NSF) Grant ATM-0645784 (Physical and Dynamic Meteorology Program). JDM's contribution was supported by LLNL under Contract DE-AC5207NA27344 and by the U.S. Department of Energy's Wind Energy Technologies Office. BZ's contribution was supported by the National Key R\&D Program of China under Grant 2018YFC1506802. Acknowledgement is also made to the National Center for Atmospheric Research (NCAR), which is sponsored by NSF, and to the Savio computational cluster provided by the Berkeley Research Computing program at the University of California, Berkeley (supported by the UC Berkeley Chancellor, Vice Chancellor for Research, and Chief Information Officer) for computing time used in this research. We also thank James Neher for updating DRM for WRF 3.8.1. Finally, a sincere thank you to the reviewers for their careful reading and thoughtful review of the first submission of this manuscript.

\section{REFERENCES}

Beare, R. J., 2014: A length scale defining partially-resolved boundary-layer turbulence simulations. Bound.Layer Meteor., 151, 39-55, https://doi.org/10.1007/s10546-013-9881-3.

Carper, M. A., and F. Porté-Agel, 2004: The role of coherent structures in subfilter-scale dissipation of turbulence measured in the atmospheric surface layer. J. Turbul., 5, 32-32, https:// doi.org/10.1088/1468-5248/5/1/040.

Ching, J., R. Rotunno, M. LeMone, A. Martilli, B. Kosovic, P. A. Jimenez, and J. Dudhia, 2014: Convectively induced secondary 
circulations in fine-grid mesoscale numerical weather prediction models. Mon. Wea. Rev., 142, 3284-3302, https://doi.org/10.1175/ MWR-D-13-00318.1.

Chow, F. K., R. L. Street, M. Xue, and J. H. Ferziger, 2005: Explicit filtering and reconstruction turbulence modeling for largeeddy simulation of neutral boundary layer flow. J. Atmos. Sci., 62, 2058-2077, https://doi.org/10.1175/JAS3456.1.

Clarke, R., A. Dyer, R. Brook, D. Reid, and A. Troup, 1971: The Wangara Experiment: Boundary layer data. CSIRO Division of Meteorological Physics Tech. Rep. 19, CSIRO, Aspendale, Australia, $362 \mathrm{pp}$.

de Roode, S. R., P. G. Duynkerke, and H. J. J. Jonker, 2004: Large-eddy simulation: How large is large enough? J. Atmos. Sci., 61, 403-421, https://doi.org/10.1175/1520-0469(2004) 061<0403:LSHLIL>2.0.CO;2.

Efstathiou, G. A., and R. J. Beare, 2015: Quantifying and improving sub-grid diffusion in the boundary-layer grey zone. Quart. J. Roy. Meteor. Soc., 141, 3006-3017, https://doi.org/ 10.1002/qj.2585.

,,-- S. Osborne, and A. P. Lock, 2016: Grey zone simulations of the morning convective boundary layer development. J. Geophys. Res. Atmos., 121, 4769-4782, https://doi.org/ 10.1002/2016JD024860.

Garcia, M., M. Özdogan, and P. A. Townsend, 2014: Impacts of forest harvest on cold season land surface conditions and land-atmosphere interactions in northern Great Lakes states. J. Adv. Model. Earth Syst., 6, 923-937, https://doi.org/10.1002/2014MS000317.

Germano, M., U. Piomelli, P. Moin, and W. H. Cabot, 1991: A dynamic subgrid-scale eddy viscosity model. Phys. Fluids A: Fluid Dyn., 3, 1760-1765, https://doi.org/10.1063/1.857955.

Gullbrand, J., and F. K. Chow, 2003: The effect of numerical errors and turbulence models in large-eddy simulations of channel flow, with and without explicit filtering. J. Fluid Mech., 495, 323-341, https://doi.org/10.1017/S0022112003006268.

Honnert, R., V. Masson, and F. Couvreux, 2011: A diagnostic for evaluating the representation of turbulence in atmospheric models at the kilometric scale. J. Atmos. Sci., 68, 3112-3131, https://doi.org/10.1175/JAS-D-11-061.1.

Khani, S., and M. L. Waite, 2014: Buoyancy scale effects in largeeddy simulations of stratified turbulence. J. Fluid Mech., 754, 75-97, https://doi.org/10.1017/jfm.2014.381.

- , and - , 2015: Large eddy simulations of stratified turbulence: The dynamic Smagorinsky model. J. Fluid Mech., 773, 327-344, https://doi.org/10.1017/jfm.2015.249.

Kirkil, G., J. Mirocha, E. Bou-Zeid, F. K. Chow, and B. Kosović, 2012: Implementation and evaluation of dynamic subfilter-scale stress models for large-eddy simulation using WRF. Mon. Wea. Rev., 140, 266-284, https://doi.org/10.1175/MWR-D-11-00037.1.

Klose, M., and Y. Shao, 2013: Large-eddy simulation of turbulent dust emission. Aeolian Res., 8, 49-58, https://doi.org/10.1016/ j.aeolia.2012.10.010.

Kurowski, M. J., and J. Teixeira, 2018: A scale-adaptive turbulent kinetic energy closure for the dry convective boundary layer. J. Atmos. Sci., 75, 675-690, https://doi.org/10.1175/JAS-D16-0296.1.

Lean, H. W., P. A. Clark, M. Dixon, N. M. Roberts, A. Fitch, R. Forbes, and C. Halliwell, 2008: Characteristics of highresolution versions of the Met Office Unified Model for forecasting convection over the United Kingdom. Mon. Wea. Rev., 136, 3408-3424, https://doi.org/10.1175/ 2008MWR2332.1.

Lilly, D., 1962: On the numerical simulation of buoyant convection. Tellus, 14, 148-172, https://doi.org/10.3402/tellusa.v14i2.9537.
_ 1967: The representation of small-scale turbulence in numerical simulation experiments. Proc. IBM Scientific Computing Symp. on Environmental Sciences, Yorktown Heights, NY, IBM, 195-210.

Liu, G., J. Sun, and L. Yin, 2011: Turbulence characteristics of the shear-free convective boundary layer driven by heterogeneous surface heating. Bound.-Layer Meteor., 140, 57-71, https:// doi.org/10.1007/s10546-011-9591-7.

Mirocha, J. D., and K. A. Lundquist, 2017: Assessment of vertical mesh refinement in concurrently nested large-eddy simulations using the Weather Research and Forecasting Model. Mon. Wea. Rev., 145, 3025-3048, https://doi.org/10.1175/ MWR-D-16-0347.1.

_ J. K. Lundquist, and B. Kosović, 2010: Implementation of a nonlinear subfilter turbulence stress model for large-eddy simulation in the Advanced Research WRF Model. Mon. Wea. Rev., 138, 4212-4228, https://doi.org/10.1175/2010MWR3286.1.

Moeng, C.-H., P. P. Sullivan, M. F. Khairoutdinov, and D. A. Randall, 2010: A mixed scheme for subgrid-scale fluxes in cloud-resolving models. J. Atmos. Sci., 67, 3692-3705, https:// doi.org/10.1175/2010JAS3565.1.

Piotrowski, Z. P., P. K. Smolarkiewicz, S. P. Malinowski, and A. A. Wyszogrodzki, 2009: On numerical realizability of thermal convection. J. Comput. Phys., 228, 6268-6290, https://doi.org/ 10.1016/j.jcp.2009.05.023.

Porté-Agel, F., M. B. Parlange, C. Meneveau, and W. E. Eichinger, 2001: A priori field study of the subgrid-scale heat fluxes and dissipation in the atmospheric surface layer. J. Atmos. Sci., $\mathbf{5 8}$, 2673-2698, https://doi.org/10.1175/1520-0469(2001)058<2673: APFSOT $>2.0 . \mathrm{CO} ; 2$.

Schaefer-Rolffs, U., 2017: A generalized formulation of the dynamic Smagorinsky model. Meteor. Z., 26, 181-187, https:// doi.org/10.1127/metz/2016/0801.

Shi, X., H. L. Hagen, F. K. Chow, G. H. Bryan, and R. L. Street, 2018: Large-eddy simulation of the stratocumulus-capped boundary layer with explicit filtering and reconstruction turbulence modeling. J. Atmos. Sci., 75, 611-637, https://doi.org/10.1175/JAS-D-17-0162.1.

Skamarock, W., 2004: Evaluating mesoscale NWP models using kinetic energy spectra. Mon. Wea. Rev., 132, 3019-3032, https://doi.org/10.1175/MWR2830.1.

- , and Coauthors, 2008: A description of the Advanced Research WRF version 3. NCAR Tech. Note NCAR/TN-475+STR, 113 pp., https://doi.org/10.5065/D68S4MVH.

Smagorinsky, J., 1963: General circulation experiments with the primitive equations. Mon. Wea. Rev., 91, 99-164, https://doi.org/ 10.1175/1520-0493(1963)091<0099:GCEWTP>2.3.CO;2.

Stull, R. B., 1988: An Introduction to Boundary Layer Meteorology. Kluwer Academic, 666 pp.

Sullivan, P. P., J. C. McWilliams, and C.-H. Moeng, 1994: A subgrid-scale model for large-eddy simulation of planetary boundary-layer flows. Bound.-Layer Meteor., 71, 247-276, https://doi.org/10.1007/BF00713741.

Taylor, D. M., F. K. Chow, M. Delkash, and P. T. Imhoff, 2016: Numerical simulations to assess the tracer dilution method for measurement of landfill methane emissions. Waste Manage., 56, 298-309, https://doi.org/10.1016/j.wasman.2016.06.040.

Thuburn, J., H. Weller, G. K. Vallis, R. J. Beare, and M. Whitall, 2018: A framework for convection and boundary layer parameterization derived from conditional filtering. J. Atmos. Sci., 75, 965-981, https://doi.org/10.1175/JAS-D-17-0130.1.

Wong, V. C., and D. K. Lilly, 1994: A comparison of two dynamic subgrid closure methods for turbulent thermal convection. Phys. Fluids, 6, 1016-1023, https://doi.org/10.1063/1.868335. 
Wyngaard, J. C., 2004: Toward numerical modeling in the "terra incognita." J. Atmos. Sci., 61, 1816-1826, https://doi.org/ 10.1175/1520-0469(2004)061<1816:TNMITT>2.0.CO;2.

Zang, Y., R. L. Street, and J. R. Koseff, 1993: A dynamic mixed subgrid-scale model and its application to turbulent recirculating flows. Phys. Fluids A: Fluid Dyn., 5, 3186-3196, https://doi.org/10.1063/1.858675.

Zhou, B., and F. K. Chow, 2011: Large-eddy simulation of the stable boundary layer with explicit filtering and reconstruction turbulence modeling. J. Atmos. Sci., 68, 2142-2155, https:// doi.org/10.1175/2011JAS3693.1.

— and — 2012: Turbulence modeling for the stable atmospheric boundary layer and implications for wind energy. Flow Turbul. Combust., 88, 255-277, https://doi.org/10.1007/s10494-011-9359-7. _, and — 2014: Nested large-eddy simulations of the intermittently turbulent stable atmospheric boundary layer over real terrain. J. Atmos. Sci., 71, 1021-1039, https://doi.org/ 10.1175/JAS-D-13-0168.1.

, J. S. Simon, and F. K. Chow, 2014: The convective boundary layer in the terra incognita. J. Atmos. Sci., 71, 2545-2563, https://doi.org/10.1175/JAS-D-13-0356.1.

, M. Xue, and K. Zhu, 2017: A grid-refinement-based approach for modeling the convective boundary layer in the gray zone: A pilot study. J. Atmos. Sci., 74, 3497-3513, https://doi.org/ 10.1175/JAS-D-16-0376.1.

,-- , and — 2018: A grid-refinement-based approach for modeling the convective boundary layer in the gray zone: Algorithm implementation and testing. J. Atmos. Sci., 75, 1143-1161, https://doi.org/10.1175/JAS-D-17-0346.1.

Zhu, P., B. A. Albrecht, V. P. Ghate, and Z. Zhu, 2010: Multiplescale simulations of stratocumulus clouds. J. Geophys. Res., 115, D23201, https://doi.org/10.1029/2010JD014400. 